\title{
Primers and polymerase chain reaction conditions for DNA barcoding teleost fish based on the mitochondrial cytochrome $b$ and nuclear rhodopsin genes
}

\author{
Rafael G. Sevilla ${ }^{1}$, Amalia Diez ${ }^{1}$, Michael Norén ${ }^{2}$, Olivier Mouchel $^{3}$, Marc Jérôme ${ }^{3}$, Véronique \\ Verrez-Bagnis ${ }^{3}$, Hilde van Pelt ${ }^{4}$, Laurence Favre-Krey ${ }^{5}$, Grigorios Krey ${ }^{5}$, The FishTrace \\ Consortium $^{6}$ and José M. Bautista ${ }^{1^{\star}}$
}

\begin{abstract}
1Department of Biochemistry and Molecular Biology IV, Universidad Complutense de Madrid (UCM), Facultad de Veterinaria, Madrid, Spain

2 Swedish Museum of Natural History, Frescativaegen 40, PO Box 50007, SE-104 05, Stockholm, Sweden 3French Research Institute for the Exploitation of the Sea (IFREMER), Centre de Nantes Rue de l'île d'Yeu, 44311, Nantes Cedex 3, France

4Netherlands Institute for Fisheries Research, Haringkade 1, 1970 AB IJmuiden, The Netherlands

5National Agricultural Research Foundation-Fisheries Research Institute, Nea Peramos, Kavala, GR-64007, Greece

6The FishTrace Consortium (www.fishtrace.org) comprises 53 members from the following institutions: University Complutense of Madrid; Joint Research Centre of the European Commission; Swedish Museum of Natural History; Canarian Institute of Marine Sciences; French Research Institute for the Exploitation of the Sea; Netherlands Institute for Fisheries Research; Natural History Museum of Funchal; Natural History Museum of Tenerife; Fisheries Research Institute of Kavala; and National Natural History Museum of Paris
\end{abstract}

${ }^{*}$ ) Corresponding author at: Department of Biochemistry and Molecular Biology IV, Universidad Complutense de Madrid, Facultad de Veterinaria, Ciudad Universitaria, 28040 Madrid, Spain. Email: imbau@vet.ucm.es

\begin{abstract}
:
This report describes a set of 21 polymerase chain reaction primers and amplification conditions developed to barcode practically any teleost fish species according to their mitochondrial cytochrome $\mathrm{b}$ and nuclear rhodopsin gene sequences. The method was successfully tested in more than 200 marine fish species comprising the main Actinopterygii family groups. When used in phylogenetic analyses, its combination of two genes with different evolutionary rates serves to identify fish at the species level. We provide a flow diagram indicating our validated polymerase chain reaction amplification conditions for barcoding and species identification applications as well as population structure or haplotyping analyses, adaptable to high-throughput analyses.
\end{abstract}




\section{ABSTRACT}

30 This report describes a set of 21 PCR primers and amplification conditions developed to

31 barcode practically any teleost fish species according to their mitochondrial cytochrome $b$ and nuclear rhodopsin gene sequences. The method was successfully tested in more than 200 marine fish species comprising the main Actinopterygii family groups. When used in phylogenetic analyses, its combination of two genes with different evolutionary rates serves to identify fish at the species level. We provide a flow diagram indicating our validated PCR amplification conditions for barcoding and species identification applications as well as population structure or haplotyping analyses, adaptable to high-throughput analyses.

Teleosts account for $>$ 95\% of the estimated 30,000 fish species alive today (Miya et al. 2003; Nelson 2006). The unequivocal identification and classification of living organisms to the

41 species level frequently relies on genetic evidence. Specific DNA sequences act as 42 unrepeatable signatures and therefore constitute a unique DNA barcode for each species. 43 Initiatives, such as The Barcode of Life Database (www.barcodinglife.org) including The Fish 44 Barcode of Life (www.fishbol.org), use a DNA-based identification system based on a 45 relatively small fragment of the mitochondrial cytochrome $c$ oxidase subunit I (COI). This short DNA sequence provides sufficient identification labels in terms of nucleotide positions

47 (Hebert et al. 2003) to discriminate even between congeneric fish species, despite only 2\% 48 sequence divergence found in $98 \%$ of these species (Ward et al. 2005). It is nevertheless clear 49 that longer length DNA barcodes will provide more efficient identification labels. Barcode 50 efficiency can be further improved by the simultaneous use of two genes showing different 51 evolutionary rates and genomic positions. The mitochondrial cytochrome $b$ gene (cyt $b$ ) and 52 the nuclear rhodopsin gene (rhod) fulfill these requirements. The cytb gene, whose 
(1) D, direct amplification; N, nested-PCR; 1st, first nested amplification reaction using outer primers; 2nd, second nested amplification reaction using inner primers; cp cyt $b$, amplification of the complete length cyt $b$ gene (1141 bp); cyt $b-5$ ', amplification of the targeted 5' fragment of the cyt $b$ gene ( 750 bp); cyt $b-3$ ', amplification of the targeted 3' fragment of the cyt $b$ gene ( 700 bp).

${ }^{(2)} \mathrm{PCR}$ cycles provided as "temperature in ${ }^{\circ} \mathrm{C}$ - seconds" as follows:

Initial Denaturation / (Denaturation / Annealing / Extension) x Number of Cycles / Final Extension

(3) Remarks:

(a) Elongation step could be extended to 120 seconds.

(b) FishCytB-F or GluFish-F could be used as forward primer for sequencing.

(c) Polymerase used: Phusion High-Fidelity DNA polymerase (Finnzymes).

(d) Elongation step could be extended to 90 seconds.

(e) FishCytB-F and CytBI-5R are used for sequencing.

(f) THR-Fish-R and CytBI-1F could be used for sequencing.

( $g$ ) CytB-7F and TruccytB-R could be used for sequencing.

(h) Fish-seq is used for sequencing (instead of FishcytB-F).

(i) 7F-seq is used for sequencing (instead of CytB-7F).

(j) CytBI-1F and THR-Fish-R could be used for sequencing.

(k) Truccytb-R and THR-Fish2-R could be used for sequencing.

(l) GluFish-F or FishcytB-F and CytBI-2R or CytBI-3R could be used for sequencing.

( $m$ ) Hotstar Qiagen kit (Q-solutions). 
53 phylogenetic performance is comparable to that of COI (Zardoya and Meyer 1996), has been

54 widely used for identifying fish species and resolving fish phylogenies (Zardoya and Doadrio

55

56

57

58

59

60

61

62

63

64

65

66

67

68

69

70

71

72

73

74

75

76

1999; Farias et al. 2001; Chen et al. 2003; Dettai and Lecointre 2005). The intronless teleost

fish rhod gene (Venkatesh et al. 1999) provides quantitatively-equal inter-species identification labels of targeted nuclear PCR amplification products throughout its coding sequence. This gene has also been used in fish phylogenetic studies (Chen et al. 2003). In addition, the nuclear and the mitochondrial genes serve mutually as a internal phylogenetic control to validate sequences obtained from a large number of samples.

Herein, we describe the use of 21 PCR primers capable of robustly and consistently amplifying targeted DNA sequences of practically any teleost fish species, and thus generating DNA sequence collections for species identification and phylogenetic purposes. The primers, 12 specific for cytb (Table 1.A) and 9 for rhod (Table 1.C), target regions of low variability, flanking the PCR amplification areas of interest in the two genes (Fig. 1). The entire cytb coding sequence (1141 bp) can be amplified in a single reaction. However, for improved amplification efficiencies two separate reactions, one for each of the 5' ( 750 bp) and the 3' ( 700 bp) fragments, with significant overlapping between the two regions (Fig 1A), are suggested. The rhod-specific primers are used for single step amplification of $460 \mathrm{bp}$ in the gene coding sequence (Fig 1B).

1 DNA from tissue samples (mostly white muscle) obtained from fish specimens was extracted using standard phenol/chloroform procedures (Sambrook et al. 1989), a DNA isolation station (ABI PRISM ${ }^{\mathrm{TM}} 6100$ Nucleic Acid PrepStation; Applied Biosystems, Inc.), or commercial column kits (Qiagen Dneasy Kit ${ }^{\circledR}$, Qiagen Dneasy Tissue Kit ${ }^{\circledR}$ and QiAmp DNA mini kit ${ }^{\circledR}$; QIAGEN GmbH, Hilden, Germany). DNA quality was checked on $0.8 \%$ agarose gels and DNA concentration was determined using the PicoGreen ${ }^{\circledR}$ DNA quantitation kit (Molecular 
77 Probes) in a 96 multiwell microplate fluorometer reader, and a standard curve $(0.2 \mathrm{ng} / \mu \mathrm{l}$ to $78140 \mathrm{ng} / \mu \mathrm{l})$.

79 Both genes were amplified in $25 \mu$ reaction mixtures containing: $1 \mu$ DNA template 80 (concentration range $20 \mathrm{ng} / \mu \mathrm{l}$ to $30 \mathrm{ng} / \mu \mathrm{l}$ ); $1 \mathrm{X}$ PCR buffer; $0.4 \mathrm{mM}$ dNTPs; $2.5 \mathrm{mM} \mathrm{MgCl}$; 81 and 1.25 U Taq DNA polymerase. Forward and reverse primer concentrations were optimised 82 and adjusted to $0.25 \mathrm{ng} / \mu \mathrm{l}$ for cytb and $0.5 \mathrm{ng} / \mu \mathrm{l}$ for rhod. For improved amplification 83 efficiencies and automated sequencing signal quality a nested or seminested PCR step was 84 found necessary in many cases. For nested and seminested PCR, 1-2 $\mu$ l of the product from 85 the first reaction was used as template for the subsequent amplification of targeted fragments. Table 2 provides alternative PCR protocols in case of amplification failure. Details of these 87 protocols including 33 alternative amplification conditions can be found in Supplementary Table 1.

The final PCR products obtained were always of the expected length as determined by agarose gel electrophoresis. After purification, products were processed for sequencing using

91 the same forward and reverse primers employed for amplification, except the the FishcytB-F 92 and CytBI-7F amplification products that were sequenced using Fish-seq and 7F-seq, 93 respectively (Table 1.B). The optimal sequencing DNA concentration was estimated at 20 ng/ $\mu l$. PCR products were bidirectionally sequenced using an ABI 3730 capillary sequencer. Table 2 provides a flow diagram of the protocol options available using the designed primers. These protocols were tested on the complete list of species provided in FishTrace 97 (www.fishtrace.org) including 1028 teleost specimens comprising 220 species from 17 98 Actinopterigii orders (Anguilliformes, Clupeiformes, Osmeriformes, Salmoniformes, 99 Aulopiformes, Ophidiiformes, Gadiformes, Batrachoidiformes, Lophiiformes, 100 Atheriniformes, Beloniformes, Beryciformes, Zeiformes, Scorpaeniformes, Perciformes, 101 Pleuronectiformes and Tetraodontiformes), 75 families and 112 genera. 
The first option (A1) in Table 2 was successful at amplifying either of the two cytb fragments

103 or the rhod fragment in $>60 \%$ of all barcoded FishTrace species. Approximately half of the

104 remaining species were barcoded using option A2 and the rest using any of the other options.

105 Overall, the above procedure yielded $>99.9 \%$ successful amplifications. After validating the 106 sequencing data, approximately 3\% did not match the expected phylogeny, mainly due to 107 sampling (misidentified specimens) or amplification-sequencing errors. In these cases, 108 repeating the procedure using newly extracted DNA or new samples was sufficient to 109 successfully amplify and sequence the target genes.

110 In conclusion, the protocol proposed is a powerful tool for barcoding practically all teleost

111 fish species and was successfully used here to provide fully validated sequence data for the 112 FishTrace genetic catalogue (www.fishtrace.org).

\section{ACKNOWLEDGMENTS}

115 We thank S. Pérez-Benavente for skilful technical assistance. Financial support was provided 116 by the FEDER program of the MCyT-Spain (1FD97-1235-C04 MAR and CAL01-020-C3) 117 and the European Commission (FishTrace contract, QLRI-CT-2002-02755).

\section{REFERENCES}

120 Chen WJ, Bonillo C, Lecointre G (2003) Repeatability of clades as a criterion of reliability: a 121 case study for molecular phylogeny of Acanthomorpha (Teleostei) with larger number of 122 taxa. Molecular phylogenetics and evolution, 26, 262-288.

123 Dettai A, Lecointre G (2005) Further support for the clades obtained by multiple molecular 124 phylogenies in the acanthomorph bush. Comptes rendus biologies, 328, 674-689. 
125 Farias IP, Orti G, Sampaio I, Schneider H, Meyer A (2001) The cytochrome $b$ gene as a 126 phylogenetic marker: the limits of resolution for analyzing relationships among cichlid 127 fishes. Journal of molecular evolution, 53, 89-103.

128 Hebert PD, Ratnasingham S, deWaard JR (2003) Barcoding animal life: cytochrome $c$ 129 oxidase subunit I divergences among closely related species. Proceedings. Biological 130 sciences / The Royal Society, 270 Suppl 1, S96-99.

131 Miya M, Takeshima H, Endo H, et al. (2003) Major patterns of higher teleostean phylogenies: a new perspective based on 100 complete mitochondrial DNA sequences. Molecular phylogenetics and evolution, 26, 121-138.

134 Nelson JS (2006). Fishes of the World, 4th edn. John Wiley and Sons, Inc., New York.

135 Sambrook J, Fritsch EF, Maniatis T (1989). Molecular Cloning: a Laboratory Manual, 2nd 136 edn. Cold Spring Harbor Laboratory Press, New York.

137 Venkatesh B, Ning Y, Brenner S (1999) Late changes in spliceosomal introns define clades in 138 vertebrate evolution. Proceedings of the National Academy of Sciences of the United 139 States of America, 96, 10267-10271.

140 Ward RD, Zemlak TS, Innes BH, Last PR, Hebert PD (2005) DNA barcoding Australia's fish 141 species. Philosophical transactions of the Royal Society of London. Series B, Biological sciences, 360, 1847-1857.

143 Zardoya R, Meyer A (1996) Phylogenetic performance of mitochondrial protein-coding genes 144 in resolving relationships among vertebrates. Molecular biology and evolution, 13, 933145942.

146 Zardoya R, Doadrio I (1999) Molecular evidence on the evolutionary and biogeographical 147 patterns of European cyprinids. Journal of molecular evolution, 49, 227-237. 
149 Table 1.- Fish-versatile primers. (A) Primer pairs for the amplification of mitochondrial 150 cytochrome $b$ (1141 bp). (B) Primers for cytb sequencing purposes. Fish-seq and 7F-seq 151 were respectively used for sequencing FishcytB-F and CytBI-7F amplification products. (C) 152 Primers pairs for amplification of the targeted fragment in the rhodopsin nuclear gene (460 153 bp).

\begin{tabular}{lllcccc}
\hline $\mathbf{( a )}$ & \multicolumn{1}{c}{ Name $(\boldsymbol{b})$} & \multicolumn{1}{c}{ Sequence $\left(5^{\prime}-3^{\prime}\right)(\boldsymbol{b})$} & Location $(\boldsymbol{c})$ & Size $(\mathrm{bp})$ & $\% \mathrm{GC}$ & $\mathrm{Tm}\left({ }^{\circ} \mathrm{C}\right)(\boldsymbol{d})$ \\
\hline $\mathbf{1}$ & GluFish-F & AACCACCGTTGTTATTCAACTACAA & 15329 & 25 & 36.0 & 57.7 \\
$\mathbf{2}$ & FishcytB-F & ACCACCGTTGTTATTCAACTACAAGAAC & 15330 & 28 & 39.3 & 60.7 \\
$\mathbf{3}$ & CytBI-6F & TTCTCAGTAGACAACGCCACCCT & 15862 & 23 & 52.2 & 61.0 \\
$\mathbf{4}$ & CytBI-7F & CTAACCCGATTCTTTGCCTTCCACTTCCT & 15883 & 29 & 48.3 & 68.3 \\
$\mathbf{5}$ & CytBI-1F & CGATTCTTCGCATTCCACTTCCT & 15889 & 23 & 47.8 & 62.5 \\
$\mathbf{6}$ & CytBI-5R & GGTCTTTGTAGGAGAAGTATGGGTGGAA & 16018 & 28 & 46.4 & 63.5 \\
$\mathbf{7}$ & CytBI-3R & GGGGTAAAGTTGTCTGGGTCTCC & 16111 & 23 & 56.5 & 60.9 \\
$\mathbf{8}$ & CytBI-2R & GCGGGGGTAAAGTTGTCTGGGTC & 16114 & 23 & 60.9 & 65.5 \\
$\mathbf{9}$ & CytBI-4R & AGGAAGTATCATTCGGGCTTAATATG & 16159 & 26 & 38.5 & 58.9 \\
$\mathbf{1 0}$ & TruccytB-R & CCGACTTCCGGATTACAAGACCG & 16528 & 23 & 56.5 & 64.6 \\
$\mathbf{1 1}$ & THR-Fish2-R & AACCTCCGACATCCGGCTTACAAGACCG & 16528 & 28 & 57.1 & 72.1 \\
$\mathbf{1 2}$ & THR-Fish-R & ACCTCCGATCTTCGGATTACAAGACC & 16529 & 26 & 50.0 & 64.4 \\
\hline
\end{tabular}

156 (B)

\begin{tabular}{lllcccc}
\hline (a) & Name & Sequence $\left(5^{\prime}-3^{\prime}\right)$ & Location $(\boldsymbol{c})$ & Size $(\mathrm{bp})$ & $\% \mathrm{GC}$ & $\mathrm{Tm}\left({ }^{\circ} \mathrm{C}\right)(\boldsymbol{d})$ \\
\hline $\mathbf{1 3}$ & Fish-seq & CCACCGTTGTTATTCAACTACAAG & 15331 & 24 & 41.7 & 56.6 \\
$\mathbf{1 4}$ & 7F-seq & CTAACCCGATTCTTTGCCTTC & 15883 & 21 & 47.6 & 56.7 \\
\hline
\end{tabular}

157 (C)

\begin{tabular}{lllcccc}
\hline (a) & Name $(\boldsymbol{b})$ & \multicolumn{1}{c}{ Sequence $\left(5^{\prime}-3^{\prime}\right)(\boldsymbol{b})$} & Location $(\boldsymbol{e})$ & Size $(\mathrm{bp})$ & $\% \mathrm{GC}$ & $\mathrm{Tm}\left({ }^{\circ} \mathrm{C}\right)(\boldsymbol{d})$ \\
\hline $\mathbf{1 5}$ & RHO-30F: & CCNTAYGAYTAYCCNCARTAYTA & 67 & 23 & 41.3 & 53.5 \\
$\mathbf{1 6}$ & Rod-F2B: & GTCTGCAAGCCCATCAGCAACTTCCG & 415 & 26 & 57.7 & 71.0 \\
$\mathbf{1 7}$ & Rod-F2W: & AGCAACTTCCGCTTCGGTGAGAA & 430 & 23 & 52.2 & 65.1 \\
$\mathbf{1 8}$ & Rod-F2x: & AGCAACTTCCGCTTCGGCGAGAA & 430 & 23 & 56.5 & 68.8 \\
$\mathbf{1 9}$ & Rod-F2: & AGCAACTTCCGCTTCGGAGAGAA & 430 & 23 & 52.2 & 64.4 \\
$\mathbf{2 0}$ & Rod-R4n: & GGAACTGCTTGTTCATGCAGATGTAGAT & 913 & 28 & 42.9 & 63.6 \\
$\mathbf{2 1}$ & Rod-4R: & CTGCTTGTTCATGCAGATGTAGAT & 913 & 24 & 41.7 & 57.2 \\
$\mathbf{2 2}$ & Rod-5R: & GGTGGTGATCATGCAGTGGCGGAA & 937 & 24 & 58.3 & 70.7 \\
$\mathbf{2 3}$ & RHO-319R: & TTNCCRCARCAYAANGTNGT & 955 & 20 & 45.0 & 66.6 \\
\hline
\end{tabular}

159 (a): Numbers correspond to positions in Figure 1.

160 (b): Reverse primers in italics.

161 (c): Nucleotide position corresponding to the 5' position in the Oncorhynchus mykiss 162 mitochondrial genome (GenBank accession number: NC 001717). Locations given for the 163 reverse primers are based on the reverse-complementary primer sequence position.

164 (d): Tm calculated using PrimerExpress ${ }^{\mathrm{TM}} 2.0$ (Applied Biosystems).

165 (e): Nucleotide position corresponding to the 5' position in the Astyanax mexicanus genomic 166 rhodopsin gene (GenBank accession number: U12328). Locations given for the reverse 167 primers are based on the reverse-complementary primer sequence position. 
168 Table 2.- Flow diagram providing alternative PCR protocols for amplification of the target 169 fragments of the cytb and rhodopsin genes. Nested or seminested PCRs comprise two 170 reactions. The first reaction uses a pair of outer primers (designated A to D in both genes), 171 which in cytb flank the whole gene. The second reaction has specific inner primers 172 (numbered) for each gene fragment. Preferential reactions are designated with a letter 173 followed by a number e.g., for cytb-5' A1 is the first choice and B1 the third choice. (A) 174 Preferential PCR conditions (primer pair and thermocycling program) for the amplification of 175 cytb as two PCR fragments: cytb-5' and cytb-3'. (B) Preferential PCR conditions for the 176 amplification of the targeted fragment of the rhod gene.

177 (A)

178

179

180

181

182

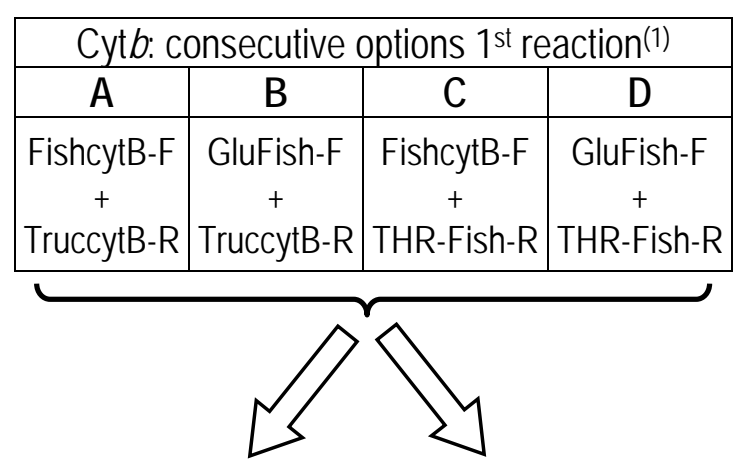

\begin{tabular}{|c|c|}
\hline \multicolumn{2}{|c|}{ Cytb-5': consecutive opt. $2^{\text {nd }}$ reaction(2) } \\
\hline $\mathbf{1}$ & $\mathbf{2}$ \\
\hline FishcytB-F & FishcytB-F \\
+ & + \\
CytBI-5R & CytBI-4R \\
\hline
\end{tabular}

\begin{tabular}{|c|c|c|c|}
\hline \multicolumn{4}{|c|}{ Cytb-3': consecutive options 2 2nd reaction(2) } \\
\hline $\mathbf{1}$ & $\mathbf{2}$ & $\mathbf{3}$ & $\mathbf{4}$ \\
\hline Cytbl-7F & Cytbl-7F & Cytbl-6F & Cytbl-6F \\
+ & + & + & + \\
THR-Fish-R & TruccytB-R & THR-Fish-R & TruccytB-R \\
\hline
\end{tabular}

(B)

\begin{tabular}{|c|c|c|c|}
\hline \multicolumn{4}{|c|}{ Rhod: consecutive options 1st reaction(3) } \\
\hline A & B & C & D \\
\hline Rod-F2B & Rod-F2B & RHO-30F & Rod-F2B \\
+ & + & + & + \\
Rod-5R & Rod-5R & RHO-319R & Rod-5R \\
\hline
\end{tabular}

PCR programs given as "temperature in ${ }^{\circ} \mathrm{C}$ - seconds" as follows: Initial Denaturation / (Denaturation / Annealing / Extension) x Number of Cycles / Final Extension: (1) 95-420 / (94-30 / 55-35 / 72-120) x35 / 72-420; (2) 95-420 / (94-30 / 55-35 / 72-45) x38 / 72-420; (3) 95-420 / (94-30 / 62-30 / 72-30) x40 / 72-420; (4) 95-420 / (94-30 / 56-30 / 72-30) x40 / 72-420. 


\section{FIGURE LEGENDS:}

198 Figure 1.- Amplification schemes: targeted gene regions and primer positions. (A) 199 Typical vertebrate cytochrome $b$ organization indicating flanking genes (tRNA ${ }^{\text {Glu }}$, 200 tRNA $^{\text {Thr }}$ ) and their nucleotides positions (15361 to 16501) within the Oncorhynchus 201 mykiss mitochondrial genome (GenBank accession number: NC 001717). The 202 relative lengths of targeted cytb-5' and cytb-3' PCR fragments are represented. 203 Detailed information on represented primer pairs is given in Table 1.A. (B) Rhodopsin 204 amplification scheme. Targeted 460bp-length fragment and primer location given as 205 that corresponding to the 5' position in the Astyanax mexicanus rhodopsin gene

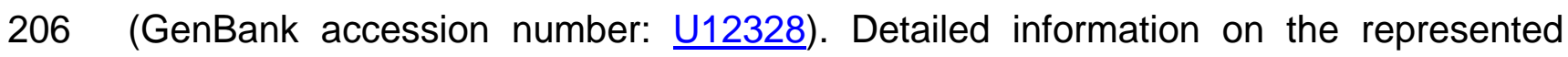
207 primer pairs is given in Table 1.B. 


\section{$209 \quad$ Figure 1}

210

211

212

(A)

(B)

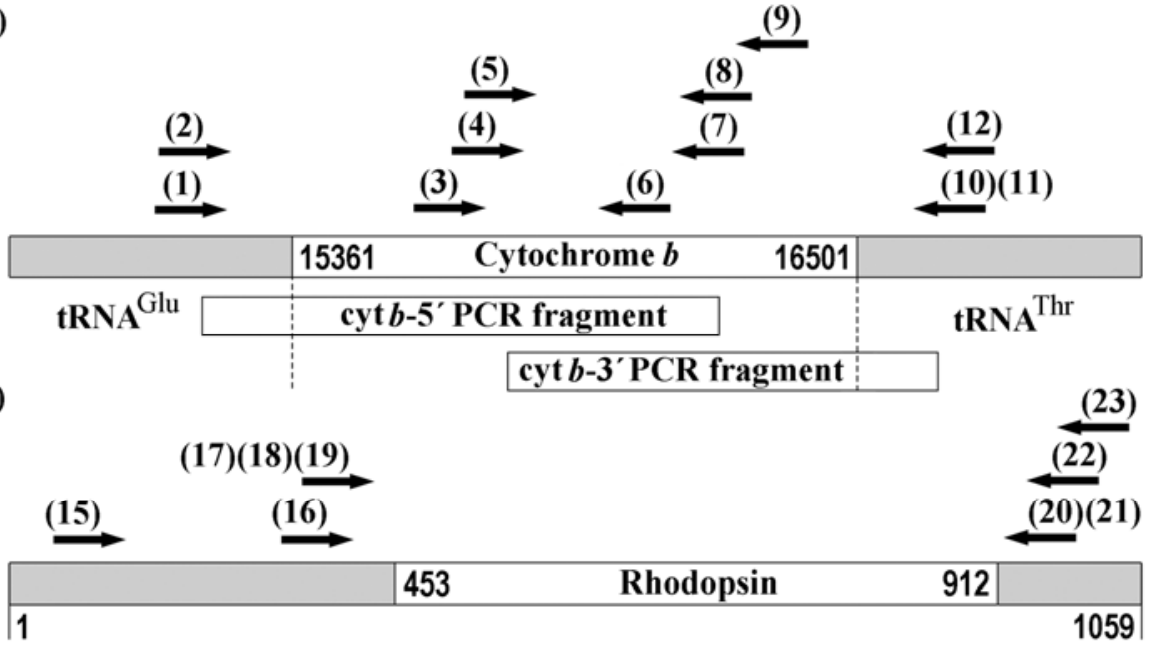


Supplementary Table 1.- PCR conditions detailing direct and nested amplifications, and alternative strategies for fish DNA barcoding. (A) Cytochrome b. (B) Rhodopsin 460bplength fragment.

(A)

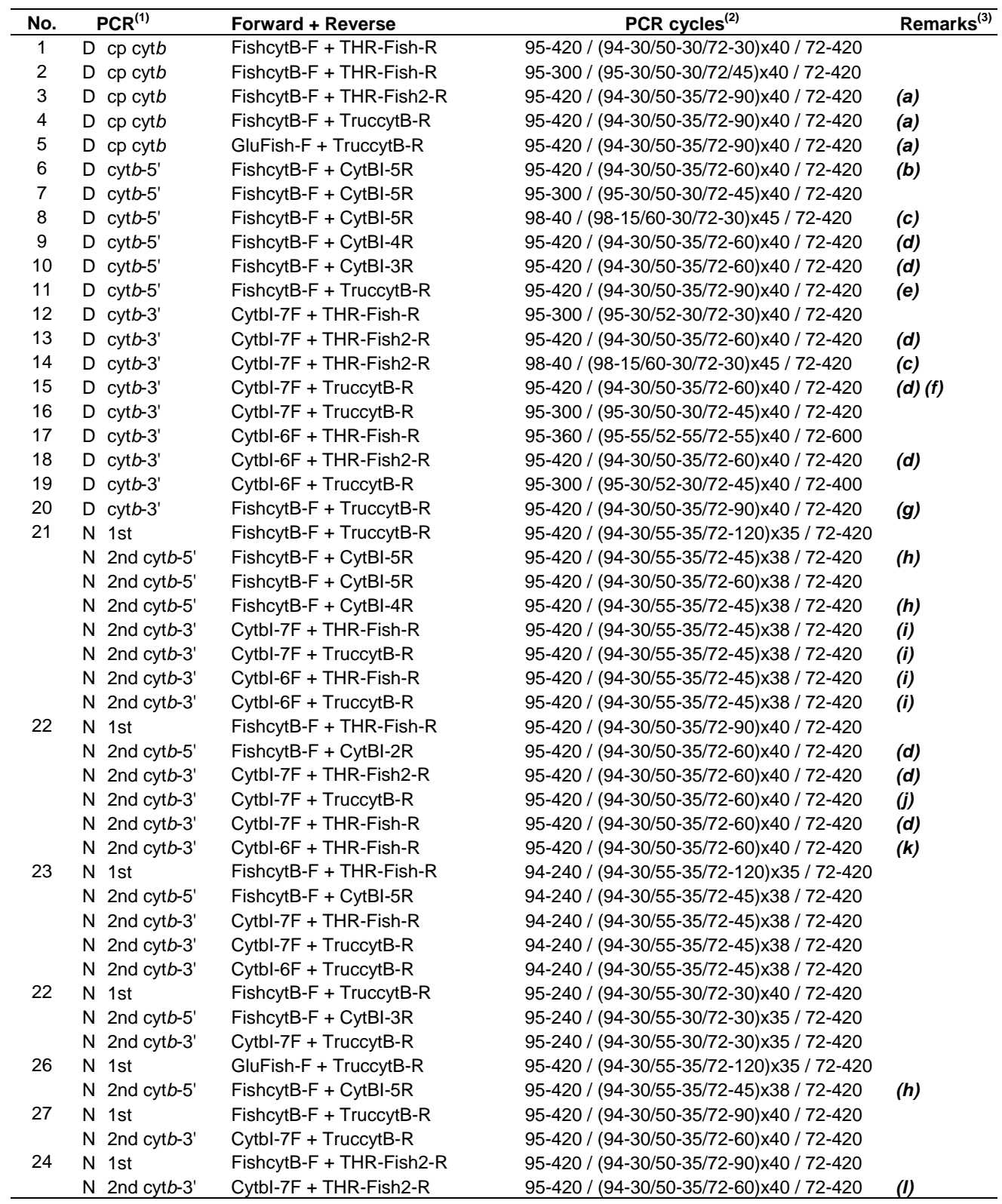

(B)

\begin{tabular}{|c|c|c|c|c|}
\hline No. & $\mathbf{P C R}^{(1)}$ & Forward + Reverse & PCR cycles $^{(2)}$ & Remarks $^{(3)}$ \\
\hline 25 & $\mathrm{D}$ & Rod-F2W + Rod-R4n & $95-240 /(94-30 / 60-30 / 72-45) \times 40 / 72-490$ & \\
\hline 26 & D & Rod-F2W + Rod-R4n & $96-60 /(96-30 / 50-30 / 60-240) \times 25 / 4-\infty$ & \\
\hline \multirow[t]{4}{*}{27} & $\mathrm{~N}$ 1st & Rod-F2B + Rod-5R & $95-420 /(94-30 / 62-30 / 72-30) \times 40 / 72-420$ & \\
\hline & $\mathrm{N}$ 2nd & Rod-F2W + Rod-R4n & $95-420 /(94-30 / 56-30 / 72-30) \times 40 / 72-420$ & \\
\hline & $N$ 2nd & Rod-F2W + Rod-R4n & $95-420 /(94-30 / 54-30 / 72-30) \times 40 / 72-420$ & \\
\hline & $\mathrm{N}$ 2nd & Rod-F2X + Rod-R4n & $95-420 /(94-30 / 56-30 / 72-30) \times 40 / 72-420$ & \\
\hline \multirow[t]{3}{*}{28} & $N 1 s t$ & Rod-F2B + Rod-5R & $95-420 /(94-30 / 60-30 / 72-30) \times 40 / 72-420$ & \\
\hline & $\mathrm{N}$ 2nd & Rod-F2W + Rod-R4n & $95-420 /(94-30 / 56-30 / 72-30) \times 40 / 72-420$ & \\
\hline & $\mathrm{N}$ 2nd & Rod-F2X + Rod-R4n & $95-420 /(94-30 / 56-30 / 72-30) \times 40 / 72-420$ & \\
\hline \multirow[t]{2}{*}{29} & $\mathrm{~N} 1 \mathrm{st}$ & RHO-30F + RHO-319R & $95-420 /(94-30 / 62-30 / 72-30) \times 40 / 72-420$ & \\
\hline & $\mathrm{N}$ 2nd & Rod-F2W + Rod-R4n & $95-420 /(94-30 / 56-30 / 72-30) \times 40 / 72-420$ & \\
\hline \multirow[t]{2}{*}{30} & $N$ 1st & Rod-F2B + Rod-5R & $94-240 /(94-30 / 60-35 / 72-120) \times 35 / 72-420$ & \\
\hline & $\mathrm{N}$ 2nd & Rod-F2 + Rod-4R & $94-240 /(94-30 / 60-35 / 72-40) \times 35 / 72-420$ & \\
\hline \multirow[t]{2}{*}{31} & $N$ 1st & Rod-F2B + Rod-5R & $95-600 /(95-55 / 54-55 / 72-55) \times 40 / 72-600$ & \\
\hline & $\mathrm{N}$ 2nd & Rod-F2 + Rod-4R & $95-600 /(95-55 / 72-55 / 72-55) \times 35 / 72-600$ & \\
\hline \multirow[t]{2}{*}{32} & $N$ 1st & Rod-F2B + Rod-5R & $95-240 /(94-30 / 55-30 / 72-30) \times 40 / 72-420$ & \\
\hline & $\mathrm{N}$ 2nd & Rod-F2 + Rod-4R & $95-240 /(94-30 / 55-30 / 72-30) \times 40 / 72-420$ & \\
\hline \multirow[t]{2}{*}{33} & $N$ 1st & Rod-F2B + Rod-5R & $95-600 /(94-55 / 56-55 / 72-55) \times 40 / 72-600$ & (m) \\
\hline & $\mathrm{N}$ 2nd & Rod-F2 + Rod-4R & $95-600 /(94-55 / 56-55 / 72-55) \times 40 / 72-600$ & (m) \\
\hline
\end{tabular}


(1) D, direct amplification; N, nested-PCR; 1st, first nested amplification reaction using outer primers; 2nd, second nested amplification reaction using inner primers; ср cytb, amplification of the complete length cytb gene (1141 bp); cytb-5', amplification of the targeted 5' fragment of the cytb gene ( 750 bp); cytb-3', amplification of the targeted 3' fragment of the cyt $b$ gene ( 700 bp).

${ }^{(2)}$ PCR cycles provided as "temperature in ${ }^{\circ} \mathrm{C}$ - seconds" as follows:

Initial Denaturation / (Denaturation / Annealing / Extension) x Number of Cycles / Final Extension

(3) Remarks:

(a) Elongation step could be extended to 120 seconds.

(b) FishCytB-F or GluFish-F could be used as forward primer for sequencing.

(c) Polymerase used: Phusion High-Fidelity DNA polymerase (Finnzymes).

(d) Elongation step could be extended to 90 seconds.

(e) FishCytB-F and CytBI-5R are used for sequencing.

( $f$ ) THR-Fish-R and CytBI-1F could be used for sequencing.

(g) CytB-7F and TruccytB-R could be used for sequencing.

(h) Fish-seq is used for sequencing (instead of FishcytB-F).

(i) 7F-seq is used for sequencing (instead of CytB-7F).

(j) CytBI-1F and THR-Fish-R could be used for sequencing.

(k) Truccytb-R and THR-Fish2-R could be used for sequencing.

(l) GluFish-F or FishcytB-F and CytBI-2R or CytBI-3R could be used for sequencing.

(m) Hotstar Qiagen kit (Q-solutions). 